International Journal of Statistics and Analysis.

ISSN 2248-9959 Volume 1, Number 4 (2011), pp. 417-439

(C) Research India Publications

https://dx.doi.org/10.37622/IJSA/1.4.2011.417-441

\title{
Generation of Model Life Tables for the Major States of India
}

\author{
Labananda Choudhury ${ }^{1}$ and Rajan Sarma ${ }^{2}$ \\ ${ }^{1}$ Department of Statistics, Gauhati University, Guwahati- 781014, Assam, India \\ ${ }^{2}$ Department of Statistics, Darrang College, Tezpur-784001, Assam, India
}

\begin{abstract}
Life tables are, in essence, one form of combining age specific mortality rates of a population into a single statistical model. In India, registration of deaths is not satisfactory and the conventional way of constructing life tables from age specific deaths cannot be adopted. Sample registration system (SRS) provides life tables only for the country and the major states. Life tables for the districts of India are not available. However, districts in India are large enough both in population and geographical sizes; some even have a population larger than some smaller European countries. At the time when 2001 census was carried out, India has 28 states and 7 union territories with 593 districts. It may be immensely useful for health and planning purposes to have life tables for these districts.

The present study proposes a new method of generating model life tables for India and its major states. The life tables of the major states obtained from the model life tables are satisfactorily closer to the corresponding SRS life tables. The life table of a district can be obtained from the model life table of the state to which it belongs.
\end{abstract}

Keywords: Life Table, Life expectancy at birth, Model Life Table, India, Districts.

\section{Introduction}

A life table is designed essentially to measure mortality, but has been employed in various fields by various specialists. It is used by public health workers, demographers, actuaries, and many others in studies of longevity, fertility, migration, population growth, etc. as well as in studies of widowhood, orphanhood, length of married life, length of working life and length of disability free life. In other 
applications the mortality rates in the life table are combined with other demographic data into a complex model which measures the combined effect of mortality and changes in one or more socio-economic characteristics ${ }^{[1]}$.

The life expectancy at birth $\left(\mathrm{e}_{0}{ }^{0}\right)$, the final output of a life table, is the average number of years a new born of a particular place can expect to live if the current age schedule of mortality prevails. It is usually considered as an important indicator of the mortality level of a population ${ }^{[2]}$.

The conventional way of constructing life tables is by using the information on age specific deaths provided by a vital registration system. Unfortunately, in India, the registration of deaths is not satisfactory and this procedure cannot be adopted ${ }^{[3,4]}$. Sample Registration System (SRS) is the main source of reliable information on vital statistics for the country and its constituent states. However, possibly due to its sample size problems, SRS has not gone beyond the 16 major states in estimating the life expectancy at birth $\left(\mathrm{e}_{0}{ }^{0}\right)$ and the construction of the life tables. The life tables for the sub-state units like the districts are not available.

A number of indirect techniques are available in the literature of mortality estimation in the absence of observed age specific deaths ${ }^{[5]}$. Most of these methods are not applicable at the sub state level of India as either one or more required data elements are not available or the underlying assumptions are not fulfilled. In cases where the required data are available, the results obtained from the application of these methods reveal inconsistencies which lead to suspect the quality of data or the applicability of the methods at the sub state level.

However, life expectancy at birth for the districts of India can indirectly be estimated by regression method using estimated infant mortality rates ( $\mathrm{q}(1)$ or ${ }_{1} \mathrm{q}_{0}$ ) and the proportion of persons above 65 years of age $\left(\mathrm{P}\left(65^{+}\right)\right)$as independent variables [6].

Generally, after estimation of $\mathrm{e}_{0}{ }^{0}$ for a district of India, the usual procedure to construct the life table, is to look for a suitable model life table, particularly, the United Nations South Asian Model, select two life tables of this model corresponding to two consecutive $\mathrm{e}_{0}{ }^{0}$ within which the estimated $\mathrm{e}_{0}{ }^{0}$ of the district falls and interpolate the values of the life table functions. It is often found that such interpolated life tables differ considerably with the SRS life tables of many states of India. For instance, the SRS life tables $\left({ }_{n} q_{x}\right.$ and $e_{x}{ }^{0}$ portion) of Tamil Nadu (RuralFemale: 1993-97), and Orissa (Urban-Female: 2001-05) differ considerably with the interpolated South Asian Model life tables (Tables1-2, Figures 1-2). Another problem of using the model life table is that it will yield the same life table for the different districts of different states having the same $\mathrm{e}_{0}{ }^{0}$. For example, the $\mathrm{e}_{0}{ }^{0}$ of a developed district of Assam may be same to that of a less developed district of say, Kerala or Punjab, and the life tables constructed from a model one with the given $\mathrm{e}_{0}{ }^{0}$ will be identical. But these states generally differ in population structure, religion, culture and the people are from different racial and ethnic background. With so many differences in socio-cultural and demographic factors it is unlikely that districts of different states will have the same mortality pattern even if they have the same $\mathrm{e}_{0}{ }^{0}$. For example, consider the SRS life tables $\left(\mathrm{n}_{\mathrm{x}}\right.$ and $\mathrm{e}_{\mathrm{x}}{ }^{0}$ portion) of Uttar Pradesh (2000-04) and Assam (1989-93) for urban female; Haryana and Tamil Nadu (1993-97) for rural 
female; though their $\mathrm{e}_{0}{ }^{0}$ 's are same, the life table functions ${ }_{\mathrm{n}} q_{\mathrm{x}}$ 's (probability of dying in the age group $x$ to $x+n$ ) and $e_{x}{ }^{0}$ 's (life expectancy at age $x$ ) differ (Table 3, Figures 3-4). This necessitates the search for an alternative method.

Office of the Registrar General of India produces SRS based abridged life tables for India and its major states starting from 1970-75 onwards almost at regular intervals ${ }^{[7]}$. It is found from these life tables that $\mathrm{e}_{\mathrm{x}}{ }^{0}$ and ${ }_{\mathrm{n}} q_{\mathrm{x}}$ can be obtained from $\mathrm{e}_{0}{ }^{0}$ by different regression equations for different x's. Clubbing all these life tables and carrying out regression analysis, a set of one parameter model life tables can be generated for India which can further be used to estimate life tables for the districts with $\mathrm{e}_{0}{ }^{0}$ as the only input. But, this does not eliminate the problem of having identical life tables for the districts of different states with the same $\mathrm{e}_{0}^{0}$. This problem may be reduced by generating separate model life tables for India and its 16 major states from the SRS based abridged life tables. The life table of a district can be obtained from the model life table of the state to which the district belongs. The districts of the same state with same $\mathrm{e}_{0}{ }^{0}$ will, Of course, have partly identical life tables (from age 5 and above), but it will, at least, bring down the problem of overlooking the macro (state) level heterogeneity to micro (district) level heterogeneity.

\section{Objectives}

The present study proposes a new method of constructing life tables by estimating the life table functions $\mathrm{e}_{\mathrm{x}}{ }^{0}$, $s$ and ${ }_{\mathrm{n}} \mathrm{q}_{\mathrm{x}}$ 's from the estimated $\mathrm{e}_{0}{ }^{0}$ using the regression method of curve estimation for India and its major states. The generated life tables of India serve as model life tables for the smaller states and the union territories and the generated life tables of the major states serve as the model life tables for their constituent districts.

\section{Methods and Materials}

Adekola ${ }^{[8]}$ presented a mathematical life expectancy model for a non homogeneous population, which depends on the life expectancy at birth and age of the population:

$$
L(A)=L(0)(1-\beta A)^{\theta} \quad 0 \leq A \leq \varpi, \quad \theta \geq 0
$$

Where $L(A)$ and $L(0)$ are non-negative constants and denote the life expectancy at age $\mathrm{A}$ and at birth respectively, and $\theta$ and $\beta$ are constants representing the scale and shape parameters respectively, $\varpi$ is the maximum age lived in the population.

Following Adekola's model, we have checked how the $\mathrm{e}_{\mathrm{x}}^{0}$ 's changes from $\mathrm{e}_{0}^{0}$ in SRS life tables of India for different periods and found that the percentage change in $\mathrm{e}_{\mathrm{x}}{ }^{0}(\mathrm{x}=1,5,10, \ldots, 70)$ from $\mathrm{e}_{0}^{0}$ is almost constant in different periods and the difference, if any, is not more than 1\% (Table 4). Similar situations are found in the SRS life tables of the major states of India.

SRS data covering a period of 30 years from 1970-75 to 2001-05 for India and its major states (Government of India, 1970-75 to 2001-05) were used to check for the existence of appropriate functional relationship (Mth) between $\mathrm{e}_{\mathrm{x}}{ }^{0}$ and $\mathrm{e}_{0}{ }^{0}$. It is found 
that different functional relationships exist between $\mathrm{e}_{\mathrm{x}}{ }^{0}$ and $\mathrm{e}_{0}{ }^{0}$ for different $\mathrm{x}$ 's with very high $\mathrm{R}^{2}$ (Rsq). Generally, a linear (LIN) or a quadratic (QUA) relationship will satisfactorily estimate the $\mathrm{e}_{\mathrm{x}}{ }^{0} \mathrm{~s}$ (Table 5).

In the Coale Demeny ${ }^{[9]}$ regional model life tables four patterns observed in the 192 life tables by sex were identified. For each of these patterns coefficients of linear equations relating to ${ }_{n} q_{x}$ and $e_{10}{ }^{0}$ were obtained by least square regression. From the equations thus established, it was simple to derive a complete set of ${ }_{n} q_{x}$ values and, therefore, a model life table from any given value of $\mathrm{e}_{10}{ }^{0[5]}$.

Following the above procedure, the SRS data covering 30 years from 1970-75 to 2001-05 were used to estimate the curve of ${ }_{n} q_{x}$ from $e_{0}{ }^{0}$ instead of $e_{10}{ }^{0}$ (as we have only the estimated $\mathrm{e}_{0}{ }^{0}$ for the districts). It is found that ${ }_{n} \mathrm{q}_{\mathrm{x}}$ 's are also related to $\mathrm{e}_{0}{ }^{0}$ by different functions (linear, quadratic or cubic (CUB)) for different x's with very high $\mathrm{R}^{2}$ (Table 6). We select the function with the maximum $\mathrm{R}^{2}$ and calculate the ${ }_{n} \mathrm{q}_{\mathrm{x}}$ 's. If for some $x$, the calculated ${ }_{n} q_{x}$ results in some negative value or the ${ }_{n} q_{x}$ curve shows some irregularities we take the function with the next highest $\mathrm{R}^{2}$ and so on till all ${ }_{n} q_{x}$ values are calculated up to $\mathrm{x}=65$.

After calculating the ${ }_{n} q_{x}$ and $e_{x}{ }^{0}$, the other life table functions are calculated as:

$$
\begin{aligned}
& d_{x}=q_{x} l_{x} \text { for } x=1,5, \ldots 65 \text {, and } l_{0}=100000 \\
& l_{x+n}=l_{x}-d_{x} \\
& { }_{1} L_{0}=0.276 l_{0}+0.724 l_{1} \\
& { }_{4} L_{1}=0.034 l_{0}+1.184 l_{1}+2.782 l_{5} \\
& { }_{5} L_{5}=-0.003 l_{0}+2.242 l_{5}+2.761 l_{10}
\end{aligned}
$$

The values of ${ }_{5} L_{x}$ from ages 10 and above are obtained as ${ }_{5} L_{x}=T_{x}-T_{x+5}$ and

$$
T_{x}=\frac{e_{x}{ }^{0}}{l_{x}} .
$$

It is to be noted that ${ }_{1} q_{0}$ and ${ }_{4} q_{1}(=q(5)-q(1))$ values for the districts are estimated from 2001 census data using Trussell's variant of the Brass method and used in estimating $\mathrm{e}_{0}{ }^{0}$ for the districts ${ }^{[6]}$ and are generally different for districts even with same $\mathrm{e}_{0}{ }^{0}$.

\section{Results and Discussion}

From the life table functions generated from SRS life tables of India a set of life tables corresponding to different $\mathrm{e}_{0}{ }^{0}$ 's can be derived. The life table for India generated from the SRS $\mathrm{e}_{0}{ }^{0}$ of different periods and the corresponding SRS life tables are compared and found to be satisfactorily closer to each other. For instance, the generated life table of India corresponding to the SRS $\mathrm{e}_{0}{ }^{0}$ of 2001-05 (=63.2) well approximates the SRS life table of India for that period (Table 7, Fig 5).

It is also seen that SRS life table of a state whose $\mathrm{e}_{0}{ }^{0}$ is not far away from the range of the $\mathrm{e}_{0}{ }^{0}$ 's of India (i.e., 48.9 in 1970-75 and 63.2 in 2001-05) is almost 
identical with the generated life table of India corresponding to that $\mathrm{e}_{0}{ }^{0}$. For example, the generated life table of India corresponding to $\mathrm{e}_{0}{ }^{0}=58.7$ which is the life expectancy at birth for Assam (Person) during 2001-05 (SRS), is almost identical with the SRS Life table of Assam for that period (Table 8, Figure 6). The generated life table of India corresponding to $\mathrm{e}_{0}{ }^{0}=59.6$ which is the life expectancy at birth of Uttar Pradesh (person) during 2000-04 (SRS), is almost identical with the SRS life table of Uttar Pradesh for 2000-04 (Table 9, Figure 7), etc. Thus, the set of the generated life tables of India for different $\mathrm{e}_{0}{ }^{0}$ 's acts as Model life tables.

The problem arises with the states whose $\mathrm{e}_{0}{ }^{0}$ is outside the range of $\mathrm{e}_{0}{ }^{0}$ of India. In that case the generated life table may show some unacceptable results. Therefore, the generated life table for India may be used only to obtain the life tables of the states and union territories for which SRS life tables were not available and whose estimated $\mathrm{e}_{0}{ }^{0}$ is not too far from the range of $\mathrm{e}_{0}{ }^{0}$ of India. The life tables for the districts of the major states (whose SRS life tables are available) may be constructed by generating separate life tables for these states by exploiting the relationship of the life table functions with $\mathrm{e}_{0}{ }^{0}$ found from the SRS life tables of these states. As the life table of a constituent state of India can be obtained from the generated life tables of India corresponding to the $\mathrm{e}_{0}{ }^{0}$ of that state, the life table of a district of a particular state can be obtained from the generated life tables of that state corresponding to the estimated $\mathrm{e}_{0}{ }^{0}$ of that district.

In the SRS life tables sometimes a few irregularities are found in the ${ }_{n} q_{\mathrm{x}}$ values. For example, in the SRS life tables of Kerala, ${ }_{1} q_{0}$ values shows a decreasing trend from 0.04233 in 1970-75 to 0.00264 in 1997-2001. Then it jumps to 0.00875 in 20002004 and 0.00606 in 2001-2005. Such irregularities result in a very low $\mathrm{R}^{2}$ in the regression and may distort the curve. Elimination of such irregular data points from the regression analysis increases the $\mathrm{R}^{2}$ considerably. Thus, whenever such a situation is encountered, data points responsible for the irregularities are to be eliminated at the cost of degrees of freedom.

For the sake saving space, instead of the Model life tables we are presenting the coefficients of the curve fit of $\mathrm{e}_{\mathrm{x}}{ }^{0}$ and ${ }_{\mathrm{n}} q_{\mathrm{x}}$ with $\mathrm{e}_{0}{ }^{0}$ as the independent variable, for $\mathrm{a}$ few selected major states belonging to different zones of India viz., Himachal Pradesh (North), Assam (North East), Kerala (South), Uttar Pradesh (Central), West Bengal (East), Gujarat (West) (Table 10). Using these coefficients, $\mathrm{e}_{\mathrm{x}}{ }^{0}$ and ${ }_{\mathrm{n}} q_{\mathrm{x}}$ portion of the life table can be completed for different age groups from a given $\mathrm{e}_{0}{ }^{0}$ of the state or any of its districts and the whole abridged life table can be completed using the formulas as given above.

In Table 11 (1-6) (Figures 8(1)-8(6)), the life tables generated by using the equations of the curve fit and 2001-05 SRS $\mathrm{e}_{0}^{0}$ of the selected major states and the SRS life table (the ${ }_{\mathrm{n}} q_{\mathrm{x}}$ and $\mathrm{e}_{\mathrm{x}}{ }^{0}$ portion only) of these states for that period are presented. 
Table 1: SRS (Female-Rural: 1993-97) and South Asian Model Life Tables (Interpolated) for Tamil Nadu (qx and ex portion):

\begin{tabular}{|c|c|c|c|c|}
\hline & \multicolumn{2}{|c|}{ SRS } & \multicolumn{2}{c|}{ South Asian Model } \\
\hline Age & $\mathrm{qx}$ & $\mathrm{e}_{\mathrm{x}}{ }^{0}$ & ${ }_{\mathrm{n}} \mathrm{q}_{\mathrm{x}}$ & $\mathrm{e}_{\mathrm{x}}{ }^{0}$ \\
\hline 0 & 0.05375 & 63.3 & 0.080055 & 63.30 \\
\hline 1 & 0.01541 & 65.9 & 0.040408 & 67.78 \\
\hline 5 & 0.00703 & 62.9 & 0.008606 & 66.58 \\
\hline 10 & 0.00499 & 58.3 & 0.003748 & 62.13 \\
\hline 15 & 0.01015 & 53.6 & 0.005136 & 57.18 \\
\hline 20 & 0.01148 & 49.1 & 0.006186 & 52.64 \\
\hline 25 & 0.01282 & 44.6 & 0.007118 & 47.95 \\
\hline 30 & 0.0139 & 40.2 & 0.008898 & 43.28 \\
\hline 35 & 0.01499 & 35.7 & 0.011482 & 38.64 \\
\hline 40 & 0.0204 & 31.2 & 0.015847 & 34.06 \\
\hline 45 & 0.02681 & 26.8 & 0.022862 & 29.57 \\
\hline 50 & 0.0481 & 22.5 & 0.037254 & 25.2 \\
\hline 55 & 0.06676 & 18.5 & 0.060611 & 21.07 \\
\hline 60 & 0.12121 & 14.6 & 0.099105 & 17.25 \\
\hline 65 & 0.20038 & 11.2 & 0.153409 & 13.86 \\
\hline 70 & 1 & 8.4 & 1.000000 & 10.89 \\
\hline
\end{tabular}

Table 2: SRS (Female-Urban: 2001-05) and South Asian Model Life Tables (Interpolated) for Orissa (qx and ex portion):

\begin{tabular}{|c|c|c|c|c|}
\hline & \multicolumn{2}{|c|}{ SRS } & \multicolumn{2}{c|}{ South Asian Model } \\
\hline Age & $\mathrm{n}_{\mathrm{x}}$ & $\mathrm{e}_{\mathrm{x}}{ }^{0}$ & ${ }_{\mathrm{n}} \mathrm{q}_{\mathrm{x}}$ & $\mathrm{e}_{\mathrm{x}}{ }^{0}$ \\
\hline 0 & 0.06482 & 67.5 & 0.06371 & 67.50 \\
\hline 1 & 0.04422 & 71.2 & 0.02687 & 71.08 \\
\hline 5 & 0.00588 & 70.4 & 0.00564 & 69.00 \\
\hline 10 & 0.00643 & 65.8 & 0.00248 & 64.38 \\
\hline 15 & 0.00633 & 61.2 & 0.00334 & 59.53 \\
\hline 20 & 0.00777 & 56.6 & 0.004 & 54.72 \\
\hline 25 & 0.00822 & 52 & 0.00472 & 49.93 \\
\hline 30 & 0.00946 & 47.5 & 0.00604 & 45.15 \\
\hline 35 & 0.01015 & 42.9 & 0.00813 & 40.41 \\
\hline 40 & 0.01327 & 38.3 & 0.01181 & 35.72 \\
\hline 45 & 0.01937 & 33.8 & 0.01775 & 31.12 \\
\hline 50 & 0.02545 & 29.4 & 0.02977 & 26.63 \\
\hline 55 & 0.05324 & 25.1 & 0.04963 & 22.36 \\
\hline 60 & 0.08847 & 21.3 & 0.08322 & 18.39 \\
\hline 65 & 0.11274 & 18.2 & 0.13249 & 14.82 \\
\hline 70 & 1 & 15.1 & 1 & 11.67 \\
\hline \multicolumn{5}{|c|}{}
\end{tabular}


Table 3: SRS Life Tables of U.P. (2000-04) and Assam (2001-05) for female (Urban) and Haryana and Tamil Nadu (1993-97) for Female (Rural) (qx and ex portion):

\begin{tabular}{|c|c|c|c|c|c|c|c|c|}
\hline \multirow[t]{3}{*}{ Age } & \multirow{2}{*}{\multicolumn{2}{|c|}{$\begin{array}{c}\text { Uttar Pradesh(Female) } \\
\text { Urban(00-04) }\end{array}$}} & \multirow{2}{*}{\multicolumn{2}{|c|}{$\begin{array}{c}\text { Assam(F) } \\
\text { Urban(89-93) }\end{array}$}} & \multirow{2}{*}{\multicolumn{2}{|c|}{$\frac{\text { Haryna(Female) }}{\text { Rural(93-97) }}$}} & \multirow{2}{*}{\multicolumn{2}{|c|}{$\begin{array}{c}\text { Tamil Nadu(Female) } \\
\text { Rural(93-97) }\end{array}$}} \\
\hline & & & & & & & & \\
\hline & ${ }_{n} q_{x}$ & $e_{x}^{0}$ & ${ }_{n} q_{x}$ & $\mathrm{e}_{\mathrm{x}}^{0}$ & $q X$ & $\mathrm{e}_{\mathrm{x}}^{0}$ & $q X$ & $\mathrm{e}_{\mathrm{x}}^{0}$ \\
\hline 0 & 0.06252 & 64.1 & 0.06974 & 64.1 & 0.07792 & 63.3 & 0.05375 & 63.3 \\
\hline 1 & 0.05632 & 67.4 & 0.01576 & 67.9 & 0.04602 & 67.6 & 0.01541 & 65.9 \\
\hline 5 & 0.00742 & 67.3 & 0.00573 & 65.0 & 0.01173 & 66.8 & 0.00703 & 62.9 \\
\hline 10 & 0.00603 & 62.8 & 0.00451 & 60.3 & 0.00568 & 62.6 & 0.00499 & 58.3 \\
\hline 15 & 0.01030 & 58.2 & 0.00329 & 55.6 & 0.01079 & 57.9 & 0.01015 & 53.6 \\
\hline 20 & 0.01139 & 53.8 & 0.00872 & 50.8 & 0.01148 & 53.5 & 0.01148 & 49.1 \\
\hline 25 & 0.01237 & 49.4 & 0.00911 & 46.2 & 0.01168 & 49.1 & 0.01282 & 44.6 \\
\hline 30 & 0.01331 & 44.9 & 0.01198 & 41.6 & 0.01272 & 44.7 & 0.01390 & 40.2 \\
\hline 35 & 0.01395 & 40.5 & 0.01484 & 37.1 & 0.01390 & 40.2 & 0.01499 & 35.7 \\
\hline 40 & 0.01691 & 36.1 & 0.01307 & 32.6 & 0.01514 & 35.7 & 0.02040 & 31.2 \\
\hline 45 & 0.02001 & 31.6 & 0.02540 & 28.0 & 0.01962 & 31.2 & 0.02681 & 26.8 \\
\hline 50 & 0.04112 & 27.2 & 0.03688 & 23.6 & 0.03386 & 26.8 & 0.04810 & 22.5 \\
\hline 55 & 0.06286 & 23.3 & 0.09897 & 19.4 & 0.04581 & 22.7 & 0.06676 & 18.5 \\
\hline 60 & 0.10665 & 19.7 & 0.11168 & 16.3 & 0.08806 & 18.6 & 0.12121 & 14.6 \\
\hline 65 & 0.13572 & 16.7 & 0.18794 & 13.0 & 0.12894 & 15.2 & 0.20038 & 11.2 \\
\hline 70 & 1.00000 & 13.9 & 1.00000 & 10.4 & 1.00000 & 12.0 & 1.00000 & 8.4 \\
\hline
\end{tabular}

Table 4: Percentage change in $\mathrm{e}_{\mathrm{x}}{ }^{0}$ in SRS Life Tables of India for different periods.

\begin{tabular}{|c|c|c|c|c|c|c|c|c|}
\hline \multicolumn{10}{|c|}{ INDIA } \\
\hline Age & $1990-94$ & \multicolumn{2}{|c|}{$1992-96$} & \multicolumn{2}{|c|}{$2000-04$} & \multicolumn{2}{|c|}{$2001-05$} \\
\hline $\mathrm{x}$ & $\mathrm{e}_{\mathrm{x}}{ }^{0}$ & $\%$ change & $\mathrm{e}_{\mathrm{x}}{ }^{0}$ & $\%$ change & $\mathrm{e}_{\mathrm{x}}{ }^{0}$ & $\%$ change & $\mathrm{e}_{\mathrm{x}}{ }^{0}$ & $\%$ change \\
\hline 0 & 60.0 & & 60.7 & & 63.0 & & 63.2 & \\
\hline 1 & 64.1 & 0.07 & 64.9 & 0.07 & 66.5 & 0.06 & 66.6 & 0.05 \\
\hline 5 & 62.6 & 0.04 & 63.2 & 0.04 & 65.0 & 0.03 & 65.2 & 0.03 \\
\hline 10 & 58.3 & -0.03 & 58.9 & -0.03 & 60.5 & -0.04 & 60.7 & -0.04 \\
\hline 15 & 53.7 & -0.11 & 54.3 & -0.11 & 55.8 & -0.11 & 56.0 & -0.11 \\
\hline 20 & 49.2 & -0.18 & 49.8 & -0.18 & 51.2 & -0.19 & 51.5 & -0.19 \\
\hline 25 & 44.8 & -0.25 & 45.4 & -0.25 & 46.8 & -0.26 & 47.0 & -0.26 \\
\hline 30 & 40.4 & -0.33 & 41.0 & -0.32 & 42.3 & -0.33 & 42.5 & -0.33 \\
\hline 35 & 36.0 & -0.40 & 36.5 & -0.40 & 37.9 & -0.40 & 38.1 & -0.40 \\
\hline 40 & 31.6 & -0.47 & 32.1 & -0.47 & 33.5 & -0.47 & 33.7 & -0.47 \\
\hline 45 & 27.3 & -0.55 & 27.9 & -0.54 & 29.2 & -0.54 & 29.4 & -0.53 \\
\hline 50 & 23.2 & -0.61 & 23.8 & -0.61 & 25.1 & -0.60 & 25.2 & -0.60 \\
\hline 55 & 19.4 & -0.68 & 19.9 & -0.67 & 21.1 & -0.67 & 21.3 & -0.66 \\
\hline 60 & 15.9 & -0.74 & 16.4 & -0.73 & 17.6 & -0.72 & 17.7 & -0.72 \\
\hline 65 & 12.9 & -0.79 & 13.4 & -0.78 & 14.4 & -0.77 & 14.5 & -0.77 \\
\hline 70 & 10.3 & -0.83 & 10.7 & -0.82 & 11.6 & -0.82 & 11.7 & -0.81 \\
\hline
\end{tabular}


Table 5: Curve Fit of $e_{x}{ }^{\ominus}\left(E_{-} x\right)$ and $e_{\odot}{ }^{\ominus}$ (India) : Independent $-e_{\odot}{ }^{\ominus}$

$\begin{array}{ccrrrrr}\text { Dependent } & \text { Mth } & \text { Rsq } & \text { d.f. } & \text { b0 } & \text { b1 } & \text { b2 } \\ \text { E_1 } & \text { LIN } & .998 & 10 & 18.4830 & .7626 & \\ \text { E_5 } & \text { QUA } & .984 & 9 & 61.2566 & -.5330 & .0093 \\ \text { E_10 } & \text { LIN } & .970 & 10 & 30.4455 & .4710 & \\ \text { E_15 } & \text { LIN } & .970 & 10 & 27.5999 & .4414 & \\ \text { E_20 } & \text { LIN } & .965 & 10 & 25.6859 & .3986 & \\ \text { E_25 } & \text { LIN } & .959 & 10 & 18.8852 & .4382 & \\ \text { E_30 } & \text { LIN } & .965 & 10 & 17.2735 & .3916 & \\ \text { E_35 } & \text { LIN } & .953 & 10 & 14.9689 & .3565 & \\ \text { E_40 } & \text { LIN } & .939 & 10 & 11.9323 & .3342 & \\ \text { E_45 } & \text { LIN } & .937 & 10 & 7.8301 & .3315 & \\ \text { E_50 } & \text { LIN } & .928 & 10 & 4.0926 & .3255 & \\ \text { E_55 } & \text { LIN } & .861 & 10 & 5.1418 & .2444 & \\ \text { E_60 } & \text { LIN } & .868 & 10 & 1.7264 & .2431 & \\ \text { E_65 } & \text { LIN } & .763 & 10 & 2.6410 & .1776 & \\ \text { E_70 } & \text { LIN } & .720 & 10 & .2971 & .1725 & \end{array}$

Table 6: Curve Fit of $\mathrm{q}_{\mathrm{x}}\left(\mathrm{Q} \_\mathrm{x}\right)$ and $\mathrm{e}_{0}{ }^{0}$ (India):Independent- $\mathrm{e}_{0}{ }^{0}$

$\begin{array}{cccrcccc}\text { Dependent } & \text { Mth } & \text { Rsq } & \text { d.f. } & \text { b0 } & \text { b1 } & \text { b2 } & \text { b3 } \\ \text { Q_0 } & \text { LIN } & .989 & 10 & .3893 & -.0051 & & \\ \text { Q_1 } & \text { CUB } & .955 & 8 & .7353 & -.0159 & & 1.2 \mathrm{E}-06 \\ \text { Q_5 } & \text { LIN } & .990 & 10 & .0832 & -.0012 & & \\ \text { Q_10 } & \text { LIN } & .988 & 10 & .0296 & -.0004 & & \\ \text { Q_15 } & \text { LIN } & .978 & 10 & .0348 & -.0004 & & \\ \text { Q_20 } & \text { LIN } & .936 & 10 & .0440 & -.0005 & & \\ \text { Q_25 } & \text { LIN } & .974 & 10 & .0428 & -.0005 & & \\ \text { Q_30 } & \text { LIN } & .917 & 10 & .0523 & -.0006 & & \\ \text { Q_35 } & \text { LIN } & .900 & 10 & .0671 & -.0008 & & \\ \text { Q_40 } & \text { LIN } & .978 & 10 & .0900 & -.0011 & & \\ \text { Q_45 } & \text { LIN } & .970 & 10 & .1238 & -.0015 & & \\ \text { Q_50 } & \text { LIN } & .968 & 10 & .2028 & -.0025 & & \\ \text { Q_55 } & \text { QUA } & .987 & 8 & .3714 & -.0069 & 3.4 \mathrm{E}-05 & \\ \text { Q_60 } & \text { LIN } & .982 & 10 & .4142 & -.0048 & & \\ \text { Q_65 } & \text { LIN } & .964 & 10 & .5267 & -.0058 & & \end{array}$


Table 7: Generated and SRS Life Tables for India (2001-05) (Generated from SRS life tables of India):

\begin{tabular}{|c|c|c|c|c|c|c|c|c|}
\hline \multicolumn{10}{|c|}{ Generated from $\mathrm{e}_{0}{ }^{0}$} \\
\hline \multicolumn{1}{|c|}{} & \multicolumn{2}{l|}{ SRS (2001-05) } \\
\hline AGE & $\mathrm{lx}$ & $\mathrm{qx}$ & $\mathrm{dx}$ & $\mathrm{Lx}$ & $\mathrm{Tx}$ & $\mathrm{e}_{\mathrm{x}}{ }^{0}$ & $\mathrm{qx}$ & $\mathrm{e}_{\mathrm{x}}{ }^{0}$ \\
\hline 0 & 100000 & 0.06698 & 6698 & 95151 & 6320000 & 63.20 & 0.06534 & 63.2 \\
\hline 1 & 93302 & 0.03334 & 3111 & 364781 & 6221314 & 66.68 & 0.03939 & 66.6 \\
\hline 5 & 90191 & 0.00736 & 664 & 449093 & 5836931 & 64.72 & 0.00777 & 65.2 \\
\hline 10 & 89527 & 0.00432 & 387 & 443703 & 5390675 & 60.21 & 0.00529 & 60.7 \\
\hline 15 & 89140 & 0.00952 & 849 & 454912 & 4946972 & 55.50 & 0.00807 & 56.0 \\
\hline 20 & 88292 & 0.01240 & 1095 & 433368 & 4492061 & 50.88 & 0.01055 & 51.5 \\
\hline 25 & 87197 & 0.01120 & 977 & 435486 & 4058693 & 46.55 & 0.01208 & 47.0 \\
\hline 30 & 86220 & 0.01438 & 1240 & 436462 & 3623208 & 42.02 & 0.01425 & 42.5 \\
\hline 35 & 84981 & 0.01654 & 1406 & 424280 & 3186746 & 37.50 & 0.01706 & 38.1 \\
\hline 40 & 83575 & 0.02048 & 1712 & 406364 & 2762466 & 33.05 & 0.02123 & 33.7 \\
\hline 45 & 81863 & 0.02900 & 2374 & 395561 & 2356101 & 28.78 & 0.03031 & 29.4 \\
\hline 50 & 79489 & 0.04480 & 3561 & 397340 & 1960541 & 24.66 & 0.04407 & 25.2 \\
\hline 55 & 75928 & 0.07112 & 5400 & 318609 & 1563201 & 20.59 & 0.06970 & 21.3 \\
\hline 60 & 70528 & 0.11084 & 7817 & 354180 & 1244592 & 17.65 & 0.10887 & 17.7 \\
\hline 65 & 62711 & 0.16014 & 10042 & 300577 & 890412 & 14.20 & 0.15506 & 14.5 \\
\hline 70 & 52668 & 1.00000 & 52668 & 589835 & 589835 & 11.20 & 1.00000 & 11.7 \\
\hline
\end{tabular}

Table 8: Generated and SRS Life Tables for Assam (2001-05) (Generated from SRS life tables of India).

\begin{tabular}{|c|c|c|c|c|c|c|c|c|}
\hline & \multicolumn{7}{|c|}{ Assam 01-05 (Generated) } & \multicolumn{2}{c|}{ SRS (2001-05) } \\
\hline age & $\mathrm{lx}$ & $\mathrm{qx}$ & $\mathrm{dx}$ & $\mathrm{Lx}$ & $\mathrm{Tx}$ & $\mathrm{e}_{\mathrm{x}}{ }^{0}$ & $\mathrm{qx}$ & $\mathrm{e}_{\mathrm{x}}{ }^{0}$ \\
\hline 0 & 100000 & 0.08993 & 8993 & 93489 & 5870000 & 58.70 & 0.07981 & 58.7 \\
\hline 1 & 91007 & 0.04809 & 4377 & 352158 & 5755976 & 63.25 & 0.04043 & 62.8 \\
\hline 5 & 86630 & 0.01276 & 1105 & 430060 & 5396564 & 62.29 & 0.01237 & 61.4 \\
\hline 10 & 85525 & 0.00612 & 523 & 419980 & 4968425 & 58.09 & 0.00663 & 57.1 \\
\hline 15 & 85002 & 0.01132 & 962 & 423477 & 4548445 & 53.51 & 0.01272 & 52.5 \\
\hline 20 & 84039 & 0.01465 & 1231 & 433845 & 4124968 & 49.08 & 0.01514 & 48.2 \\
\hline 25 & 82808 & 0.01345 & 1114 & 402069 & 3691123 & 44.57 & 0.01563 & 43.9 \\
\hline 30 & 81694 & 0.01708 & 1395 & 406680 & 3289054 & 40.26 & 0.01711 & 39.5 \\
\hline 35 & 80299 & 0.02014 & 1617 & 399972 & 2882374 & 35.90 & 0.02299 & 35.2 \\
\hline 40 & 78682 & 0.02543 & 2001 & 389842 & 2482402 & 31.55 & 0.02397 & 30.9 \\
\hline 45 & 76681 & 0.03575 & 2741 & 377200 & 2092560 & 27.29 & 0.04365 & 26.6 \\
\hline 50 & 73940 & 0.05605 & 4144 & 355182 & 1715360 & 23.20 & 0.06388 & 22.7 \\
\hline 55 & 69795 & 0.08680 & 6058 & 340615 & 1360178 & 19.49 & 0.10285 & 19.1 \\
\hline 60 & 63737 & 0.13244 & 8441 & 297061 & 1019563 & 16.00 & 0.13364 & 16.0 \\
\hline 65 & 55296 & 0.18624 & 10298 & 253499 & 722501 & 13.07 & 0.19823 & 13.0 \\
\hline 70 & 44998 & 1.00000 & 44998 & 469002 & 469002 & 10.42 & 1.00000 & 10.6 \\
\hline
\end{tabular}


Table 9: Generated and SRS Life Tables for Uttar Pradesh (2000-04) (Generated from SRS life tables of India).

\begin{tabular}{|c|c|c|c|c|c|c|c|c|}
\hline \multicolumn{7}{|c|}{ Uttar Pradesh Generated 2000-04 } & \multicolumn{2}{c|}{ SRS 2000-04 } \\
\hline AGE & $\mathrm{lx}$ & $\mathrm{qx}$ & $\mathrm{dx}$ & $\mathrm{Lx}$ & $\mathrm{Tx}$ & $\mathrm{ex0}$ & $\mathrm{qx}$ & $\mathrm{ex0}$ \\
\hline 0 & 100000 & 0.08534 & 8534 & 93821 & 5960000 & 59.60 & 0.08716 & 59.6 \\
\hline 1 & 91466 & 0.04422 & 4045 & 354902 & 5847784 & 63.93 & 0.03891 & 64.2 \\
\hline 5 & 87421 & 0.01168 & 1021 & 434250 & 5486966 & 62.76 & 0.01045 & 62.8 \\
\hline 10 & 86400 & 0.00576 & 498 & 425112 & 5055895 & 58.52 & 0.00653 & 58.4 \\
\hline 15 & 85903 & 0.01096 & 941 & 430095 & 4630782 & 53.91 & 0.00976 & 53.8 \\
\hline 20 & 84961 & 0.01420 & 1206 & 434346 & 4200687 & 49.44 & 0.01356 & 49.3 \\
\hline 25 & 83755 & 0.01300 & 1089 & 409043 & 3766341 & 44.97 & 0.01588 & 44.9 \\
\hline 30 & 82666 & 0.01654 & 1367 & 412964 & 3357298 & 40.61 & 0.01922 & 40.6 \\
\hline 35 & 81299 & 0.01942 & 1579 & 405210 & 2944334 & 36.22 & 0.02128 & 36.4 \\
\hline 40 & 79720 & 0.02444 & 1948 & 393605 & 2539124 & 31.85 & 0.02636 & 32.1 \\
\hline 45 & 77771 & 0.03440 & 2675 & 381332 & 2145519 & 27.59 & 0.03559 & 27.9 \\
\hline 50 & 75096 & 0.05380 & 4040 & 363814 & 1764187 & 23.49 & 0.04837 & 23.8 \\
\hline 55 & 71056 & 0.08410 & 5976 & 345088 & 1400373 & 19.71 & 0.07562 & 19.9 \\
\hline 60 & 65080 & 0.12812 & 8338 & 304816 & 1055284 & 16.22 & 0.11471 & 16.3 \\
\hline 65 & 56742 & 0.18102 & 10271 & 258897 & 750468 & 13.23 & 0.17293 & 13.1 \\
\hline 70 & 46471 & 1.00000 & 46471 & 491571 & 491571 & 10.58 & 1.00000 & 10.3 \\
\hline
\end{tabular}

Table 10: Coefficients of curve Fit of $e_{x}{ }^{\ominus}$ : Independent $-e_{\odot}{ }^{\ominus}$

$\begin{array}{lllll}\text { Dependent } & \text { b0 } & \text { b1 } & \text { b2 } & \text { b3 }\end{array}$

Himachal Pradesh

$\begin{array}{lrr} & \text { E_1 } & 16.7441 \\ \text { E_5 } & 26.5504 & .6036 \\ \text { E_10 } & 26.0623 & .5396 \\ \text { E_15 } & 23.5012 & .5050 \\ \text { E_20 } & 21.2368 & .4678 \\ \text { E_25 } & 18.2941 & .4429 \\ \text { E_30 } & 15.2877 & .4202 \\ \text { E_35 } & 12.4981 & .3939 \\ \text { E_40 } & 9.3874 & .3735 \\ \text { E_45 } & 6.3571 & .3532 \\ \text { E_50 } & 4.4165 & .3188 \\ \text { E_55 } & 1.9128 & .2975 \\ \text { E_60 } & .4670 & .2635 \\ \text { E_65 } & .7086 & .2087 \\ \text { E_70 } & -.3263 & .1807\end{array}$


Dependent

b๑

b1

b2

b3

Assam

$\begin{array}{lrr}\text { E_1 } & 13.9180 & .8303 \\ \text { E_5 } & 23.7617 & .6308 \\ \text { E_10 } & 23.3085 & .5682 \\ \text { E_15 } & 20.3605 & .5404 \\ \text { E_20 } & 17.3094 & .5159 \\ \text { E_25 } & 13.0518 & .5154 \\ \text { E_30 } & 9.2427 & .5075 \\ \text { E_35 } & 6.1510 & .4867 \\ \text { E_40 } & 2.9495 & .4692 \\ \text { E_45 } & .7939 & .4346 \\ \text { E_50 } & -.9447 & .3975 \\ \text { E_55 } & -2.3354 & .3602 \\ \text { E_60 } & -2.8967 & .3140 \\ \text { E_65 } & -1.8696 & .2481 \\ \text { E_70 } & -1.0909 & .1933\end{array}$

Kerala

\begin{tabular}{|c|c|c|}
\hline & E_1 & 18.1500 \\
\hline E_5 & 28.8749 & .5548 \\
\hline E_10 & 27.4519 & .5082 \\
\hline E_15 & 23.4201 & 4967 \\
\hline E_20 & 19.6201 & . 4827 \\
\hline E_25 & 15.5692 & .4735 \\
\hline E_30 & 11.6962 & .4623 \\
\hline E_35 & 7.5712 & .4553 \\
\hline E_40 & 4.6977 & . 4314 \\
\hline E_45 & 1.9974 & . 4065 \\
\hline$E \_50$ & -1.3915 & .3938 \\
\hline E_55 & -4.9607 & .3861 \\
\hline E_60 & - 8.0157 & .3754 \\
\hline E_65 & -9.7199 & .3521 \\
\hline E_70 & -10.773 & .3245 \\
\hline
\end{tabular}

\section{Uttar Pradesh}

$\begin{array}{lll}\text { E_1 } & 20.2518 & .7351 \\ \text { E_5 } & 41.5156 & .3516 \\ \text { E_10 } & 41.3796 & .2821 \\ \text { E_15 } & 37.6442 & .2673 \\ \text { E_20 } & 33.9462 & .2537 \\ \text { E_25 } & 30.6255 & .2365 \\ \text { E_30 } & 26.8835 & .2263 \\ \text { E_35 } & 22.8246 & .2228 \\ \text { E_40 } & 18.8160 & .2182\end{array}$




$\begin{array}{rrr}\text { E_45 } & 15.7242 & .1983 \\ \text { E_50 } & 12.6440 & .1818 \\ \text { E_55 } & 10.7195 & .1488 \\ \text { E_60 } & 9.0924 & .1162 \\ \text { E_65 } & 7.9764 & .0806 \\ \text { E_70 } & 7.5071 & .0440\end{array}$

Dependent

b๑

b1

b2

b3

\section{West Bengal}

$\begin{array}{lrrr}\text { E_1 } & -98.354 & 4.6457 & -.0323 \\ \text { E_5 } & 229.840 & -6.3292 & .0587 \\ \text { E_10 } & 224.027 & -6.2016 & .0570 \\ \text { E_15 } & 208.966 & -5.8269 & .0537 \\ \text { E_20 } & 195.657 & -5.4922 & .0506 \\ \text { E_25 } & 179.339 & -5.0569 & .0466 \\ \text { E_30 } & 172.991 & -4.9686 & .0457 \\ \text { E_35 } & 168.187 & -4.9312 & .0452 \\ \text { E_40 } & 157.856 & -4.7091 & .0431 \\ \text { E_45 } & 162.263 & -4.9662 & .0450 \\ \text { E_50 } & 171.511 & -5.3681 & .0480 \\ \text { E_55 } & 155.860 & -4.9076 & .0436 \\ \text { E_60 } & 134.298 & -4.2775 & .0381 \\ \text { E_65 } & 108.045 & -3.4539 & .0309 \\ \text { E_70 } & 86.772 & -2.7797 & .0249\end{array}$

\section{Gujarat}

$\begin{array}{llrl}\text { E_1 } & 21.6989 & .7086 & \\ \text { E_5 } & 88.6366 & -1.3746 & .0156 \\ \text { E_10 } & 77.7003 & -1.0911 & .0128 \\ \text { E_15 } & 70.6763 & -.9848 & .0116 \\ \text { E_20 } & 66.4520 & -.9803 & .0115 \\ \text { E_25 } & 60.1811 & -.8930 & .0105 \\ \text { E_30 } & 51.4494 & -.7242 & .0089 \\ \text { E_35 } & 51.2093 & -.8514 & .0099 \\ \text { E_40 } & 46.6815 & -.8200 & .0094 \\ \text { E_45 } & 42.0611 & -.8019 & .0092 \\ \text { E_50 } & 39.1074 & -.7999 & .0088 \\ \text { E_55 } & 51.2385 & -1.3928 & .0142 \\ \text { E_60 } & 40.0496 & -1.0794 & .0011 \\ \text { E_65 } & 30.0388 & -.7715 & .0079 \\ \text { E_70 } & 48.4889 & -1.5284 & .0145\end{array}$


Coefficients of curve Fit of $q_{x}$ : Independent $-e_{\odot}{ }^{\ominus}$

Dependent

b०

b1

b2

b3

\section{Himachal Pradesh}

$\begin{array}{lrrr}\text { Q_0 } & .3347 & -.0042 & \\ \text { Q_1 } & 1.1871 & -.0272 & \\ \text { Q_5 } & .3150 & -.009 \odot & 6.5 \mathrm{E}-05 \\ \text { Q_10 } & .0480 & -.0007 & \\ \text { Q_15 } & .2179 & -.0062 & 4.5 \mathrm{E}-05 \\ \text { Q_20 } & .0519 & -.0 \odot \odot 7 & \\ \text { Q_25 } & .0675 & -.0009 & \\ \text { Q_30 } & .0621 & -.0008 & \\ \text { Q_35 } & .0732 & -.00 \odot 9 & \\ \text { Q_40 } & .0777 & -.00 \odot 9 & \\ \text { Q_45 } & .1495 & -.0018 & \\ \text { Q_50 } & .1539 & -.0017 & \\ \text { Q_55 } & .2579 & -.0029 & \\ \text { Q_60 } & .4427 & -.0053 & \\ \text { Q_65 } & .6520 & -.0076\end{array}$

Dependent

b๑

b1

$$
2.2 \mathrm{E}-06
$$

b2

b3

\section{Assam}

Q_0

Q_1

Q_5

Q_10

Q_15

Q_20

Q_25

Q_30

Q_35

Q_40

Q_45

Q_50

Q_55

Q_60

Q_65
.3337

.3817

.0937

.0480

.2407

.0441

.0499

.0753

.0766

.3505

.2034

.2846

.4119

.6305

.7030
- . .0044

$$
-.0083
$$

$-.0014$

- . 0007

$-.0062$

- . 0005

- . 0006

- . 0010

- . 0009

- . 0099

- . 0027

$-.0037$

$-.0054$

$-.0084$

$-.0086$
4. 1E-05

6. 8E- 07

\section{Kerala}

Q_o

Q_1

$.2950-.0038$

Q_5

.2276

$-.0030$

$.2797-.0068$

Q_10

.0415

- . 0009

4. 4E- 05

.0031

.0003

4. $8 \mathrm{E}-06$

$-4.0 \mathrm{E}-\odot 6$ 


$\begin{array}{llll}\text { Q_20 } & .1036 & -.0026 & 1.7 \mathrm{E}-05 \\ \text { Q_25 } & .1265 & -.0031 & 2.0 \mathrm{E}-05 \\ \text { Q_30 } & .0961 & -.0020 & 1.1 \mathrm{E}-05 \\ \text { Q_35 } & .0534 & -.0006 & \\ \text { Q_40 } & .0784 & -.0009 & \\ \text { Q_45 } & .1372 & -.0022 & 8.1 \mathrm{E}-06 \\ \text { Q_50 } & .1217 & -.0012 & \\ \text { Q_55 } & .2009 & -.0020 & \\ \text { Q_60 } & .2619 & -.0024 & \\ \text { Q_65 } & .8117 & -.00135 & 5.6 \mathrm{E}--05\end{array}$

Uttar Pradesh

$\begin{array}{lll}\text { Q_0 } & .3644 & -.0029 \\ \text { Q_1 } & .4402 & -.0068 \\ \text { Q_5 } & .0916 & -.0013 \\ \text { Q_10 } & .0257 & -.00 \odot 3 \\ \text { Q_15 } & .0305 & -.00 \odot 3 \\ \text { Q_20 } & .0392 & -.00 \odot 4 \\ \text { Q_25 } & .0352 & -.00 \odot 3 \\ \text { Q_30 } & .0353 & -.0003 \\ \text { Q_35 } & .0433 & -.00 \odot 4 \\ \text { Q_40 } & .0715 & -.0008 \\ \text { Q_45 } & .0988 & -.0 \odot 11 \\ \text { Q_50 } & .1679 & -.0 \odot 20 \\ \text { Q_55 } & .2443 & -.0028 \\ \text { Q_60 } & .3650 & -.0042 \\ \text { Q_65 } & .4305 & -.0042\end{array}$

Dependent

b๑

b1

b2

b3

\section{West Bengal}

$\begin{array}{lrr}\text { Q_0 } & .3517 & -.0047 \\ \text { Q_1 } & 3.2101 & .0789 \\ \text { Q_5 } & .0970 & -.0014 \\ \text { Q_10 } & .0386 & -.0005 \\ \text { Q_15 } & .0661 & -.0009 \\ \text { Q_20 } & .0646 & -.0009 \\ \text { Q_25 } & .0609 & -.0008 \\ \text { Q_30 } & .0668 & -.0009 \\ \text { Q_35 } & .0731 & -.0009 \\ \text { Q_40 } & .1210 & -.0016 \\ \text { Q_45 } & .1676 & -.0022 \\ \text { Q_50 } & .2918 & -.0039 \\ \text { Q_55 } & .2667 & -.0030 \\ \text { Q_60 } & .4420 & -.0051 \\ \text { Q_65 } & .6364 & -.0073\end{array}$




\begin{tabular}{|c|c|c|c|}
\hline \multicolumn{4}{|l|}{ Gujarat } \\
\hline Q_O & .4285 & - . .0058 & \\
\hline Q_1 & .3609 & -.0053 & \\
\hline Q_5 & .0636 & - . ๑๑९९ & \\
\hline Q_10 & .0085 & .0004 & 7.E- -06 \\
\hline Q_15 & .0334 & - . .๑๑४4 & \\
\hline Q_20 & .0471 & -.0006 & \\
\hline Q_25 & .0412 & -.0005 & \\
\hline Q_30 & .0907 & - . . 0019 & $1.5 E-05$ \\
\hline Q_35 & .0653 & -.0008 & \\
\hline Q_40 & .0895 & - .0011 & \\
\hline Q_45 & .1250 & -.0015 & \\
\hline Q_50 & .2079 & -.0026 & \\
\hline Q_55 & .2800 & -.0033 & \\
\hline Q_60 & .3944 & -.0045 & \\
\hline Q_65 & .3975 & -.0035 & \\
\hline
\end{tabular}

Table 11: Generated and SRS (2001-05) Life Tables of the major states:

Himachal Pradesh (2001-05) (Generated from SRS life tables of Himachal Pradesh)

\begin{tabular}{|c|c|c|c|c|c|c|c|c|}
\hline \multirow[t]{2}{*}{ age } & \multicolumn{5}{|c|}{ Generated } & & \multicolumn{2}{|c|}{ SRS } \\
\hline & $\mathrm{lx}$ & qX & $\mathrm{dx}$ & $\mathrm{Lx}$ & $\mathrm{Tx}$ & $\mathrm{e}_{\mathrm{x}}^{0}$ & qX & $\mathrm{e}_{\mathrm{x}}^{0}$ \\
\hline 0 & 100000 & 0.05414 & 5414 & 96080 & 6680000 & 66.80 & 0.05081 & 66.8 \\
\hline 1 & 94586 & 2591 & 2451 & 371710 & 6598628 & 69.76 & 0.03364 & 69.4 \\
\hline 5 & 92135 & 0.00385 & 354 & 459674 & 6161162 & 66.87 & 0.00374 & 67.8 \\
\hline 10 & 91781 & & 114 & 453705 & 5700289 & 2.11 & 00160 & 63.0 \\
\hline 15 & 91667 & 0.00454 & 416 & 457207 & 5246584 & 57.24 & 0.00454 & 58.1 \\
\hline 20 & 91251 & 514 & 469 & 442760 & 4789377 & 52.49 & 0.00578 & 53.3 \\
\hline 25 & 90782 & 0.00738 & 670 & 439632 & 4346617 & 47.88 & 0.00683 & 48.6 \\
\hline 30 & 90112 & 0.00866 & 780 & 439975 & 3906984 & 43.36 & 0.01025 & 44.0 \\
\hline 35 & 89331 & 0.01218 & 1088 & 436978 & 3467010 & 38.81 & 0.01312 & 39.4 \\
\hline 40 & 88243 & 0.01758 & 1551 & 433529 & 3030032 & 34.34 & 0.01991 & 34.9 \\
\hline 45 & 86692 & 0.02926 & 2537 & 432668 & 2596503 & 29.95 & 0.02612 & 30.5 \\
\hline 50 & 84155 & 0.04034 & 3395 & 404399 & 2163834 & 25.71 & 0.03434 & 26.3 \\
\hline 55 & 80761 & 0.06418 & 5183 & 393842 & 1759435 & 21.79 & 0.06256 & 22.1 \\
\hline 60 & 75577 & 0.08866 & 6701 & 356566 & 1365593 & 18.07 & 0.08171 & 18.4 \\
\hline 65 & 68877 & 0.14432 & 9940 & 316851 & 1009028 & 14.65 & 0.13460 & 14.8 \\
\hline 70 & 58936 & 1.00000 & 58936 & 692177 & 692177 & 11.74 & 1.00000 & 11.7 \\
\hline
\end{tabular}


Assam (2001-05) (Generated from SRS life tables of Assam)

\begin{tabular}{|c|c|c|c|c|c|c|c|c|}
\hline & \multicolumn{7}{|c|}{ Generated } & \multicolumn{2}{c|}{ SRS } \\
\hline age & $\mathrm{lx}$ & $\mathrm{qx}$ & $\mathrm{dx}$ & $\mathrm{Lx}$ & $\mathrm{Tx}$ & $\mathrm{e}_{\mathrm{x}}{ }^{0}$ & $\mathrm{qx}$ & $\mathrm{e}_{\mathrm{x}}{ }^{0}$ \\
\hline 0 & 100000 & 0.07459 & 7459 & 94600 & 5870000 & 58.7 & 0.07981 & 58.7 \\
\hline 1 & 92541 & 0.03364 & 3113 & 361757 & 5810914 & 62.79 & 0.04043 & 62.8 \\
\hline 5 & 89428 & 0.01152 & 1030 & 444263 & 5460863 & 61.06 & 0.01237 & 61.4 \\
\hline 10 & 88398 & 0.00411 & 363 & 423377 & 5028031 & 56.88 & 0.00663 & 57.1 \\
\hline 15 & 88034 & 0.01058 & 931 & 447713 & 4604654 & 52.31 & 0.01272 & 52.5 \\
\hline 20 & 87103 & 0.01178 & 1026 & 418340 & 4156941 & 47.72 & 0.01514 & 48.2 \\
\hline 25 & 86077 & 0.01381 & 1189 & 417051 & 3738600 & 43.43 & 0.01563 & 43.9 \\
\hline 30 & 84888 & 0.01960 & 1664 & 424167 & 3321550 & 39.13 & 0.01711 & 39.5 \\
\hline 35 & 83224 & 0.02506 & 2086 & 416799 & 2897382 & 34.81 & 0.02299 & 35.2 \\
\hline 40 & 81139 & 0.02339 & 1898 & 393958 & 2480584 & 30.57 & 0.02397 & 30.9 \\
\hline 45 & 79241 & 0.04250 & 3368 & 391972 & 2086626 & 26.33 & 0.04365 & 26.6 \\
\hline 50 & 75873 & 0.06878 & 5219 & 367751 & 1694654 & 22.34 & 0.06388 & 22.7 \\
\hline 55 & 70654 & 0.09388 & 6633 & 335425 & 1326903 & 18.78 & 0.10285 & 19.1 \\
\hline 60 & 64021 & 0.14162 & 9067 & 296764 & 991479 & 15.49 & 0.13364 & 16.0 \\
\hline 65 & 54955 & 0.20472 & 11250 & 248611 & 694714 & 12.64 & 0.19823 & 13.0 \\
\hline 70 & 43704 & 1.00000 & 43704 & 446103 & 446103 & 10.21 & 1.00000 & 10.6 \\
\hline
\end{tabular}

\section{Kerala (2001-05) (Generated from SRS life tables of Kerala)}

\begin{tabular}{|c|c|c|c|c|c|c|c|c|}
\hline \multirow{2}{*}{ age } & \multicolumn{7}{|c|}{ Generated } & \multicolumn{2}{c|}{ SRS } \\
\cline { 2 - 9 } & $\mathrm{lx}$ & $\mathrm{qx}$ & $\mathrm{dx}$ & $\mathrm{Lx}$ & $\mathrm{Tx}$ & $\mathrm{e}_{\mathrm{x}}{ }^{0}$ & $\mathrm{qx}$ & $\mathrm{e}_{\mathrm{x}}{ }^{0}$ \\
\hline 0 & 100000 & 0.01418 & 1418 & 98973 & 7390000 & 73.90 & 0.01169 & 73.9 \\
\hline 1 & 98582 & 0.00590 & 582 & 392758 & 7267741 & 73.72 & 0.00606 & 73.8 \\
\hline 5 & 98000 & 0.00177 & 174 & 489516 & 6847738 & 69.87 & 0.00215 & 70.2 \\
\hline 10 & 97827 & 0.00120 & 118 & 484636 & 6359499 & 65.01 & 0.0018 & 65.4 \\
\hline 15 & 97709 & 0.00343 & 335 & 490887 & 5874863 & 60.13 & 0.00295 & 60.5 \\
\hline 20 & 97374 & 0.00430 & 419 & 481829 & 5383976 & 55.29 & 0.00504 & 55.6 \\
\hline 25 & 96955 & 0.00663 & 643 & 485255 & 4902147 & 50.56 & 0.00643 & 50.9 \\
\hline 30 & 96312 & 0.00837 & 806 & 494466 & 4416892 & 45.86 & 0.00767 & 46.2 \\
\hline 35 & 95506 & 0.00906 & 865 & 460654 & 3922427 & 41.07 & 0.01065 & 41.6 \\
\hline 40 & 94640 & 0.01189 & 1125 & 465758 & 3461773 & 36.58 & 0.01253 & 37.0 \\
\hline 45 & 93515 & 0.01886 & 1763 & 453542 & 2996015 & 32.04 & 0.02045 & 32.4 \\
\hline 50 & 91752 & 0.03302 & 3030 & 451105 & 2542473 & 27.71 & 0.03056 & 28.0 \\
\hline 55 & 88722 & 0.04608 & 4089 & 421853 & 2091368 & 23.57 & 0.04657 & 23.8 \\
\hline 60 & 84634 & 0.08454 & 7155 & 406588 & 1669515 & 19.73 & 0.0799 & 19.9 \\
\hline 65 & 77479 & 0.11988 & 9288 & 362293 & 1262926 & 16.30 & 0.11748 & 16.4 \\
\hline 70 & 68191 & 1.00000 & 68191 & 900633 & 900633 & 13.21 & 1.00000 & 13.2 \\
\hline
\end{tabular}


Uttar Pradesh (2001-05) (Generated from SRS life tables of Uttar Pradesh)

\begin{tabular}{|c|c|c|c|c|c|c|c|c|}
\hline age & \multicolumn{7}{|c|}{ Generated } & \multicolumn{2}{c|}{ SRS } \\
\cline { 2 - 9 } & $\mathrm{lx}$ & $\mathrm{qx}$ & $\mathrm{dx}$ & $\mathrm{Lx}$ & $\mathrm{Tx}$ & $\mathrm{e}_{\mathrm{x}}{ }^{0}$ & $\mathrm{qx}$ & $\mathrm{e}_{\mathrm{x}}{ }^{0}$ \\
\hline 0 & 100000 & 0.08370 & 8370 & 93940 & 5980000 & 59.80 & 0.08627 & 59.8 \\
\hline 1 & 91630 & 0.03356 & 3075 & 358250 & 5883641 & 64.21 & 0.03910 & 64.4 \\
\hline 5 & 88555 & 0.01386 & 1227 & 439352 & 5538344 & 62.54 & 0.01025 & 63.0 \\
\hline 10 & 87328 & 0.00776 & 678 & 439834 & 5086763 & 58.25 & 0.00658 & 58.6 \\
\hline 15 & 86650 & 0.01256 & 1088 & 444358 & 4646929 & 53.63 & 0.00946 & 54.0 \\
\hline 20 & 85562 & 0.01528 & 1307 & 430659 & 4202571 & 49.12 & 0.01430 & 49.5 \\
\hline 25 & 84254 & 0.01826 & 1538 & 428851 & 3771912 & 44.77 & 0.01509 & 45.2 \\
\hline 30 & 82716 & 0.01736 & 1436 & 404954 & 3343061 & 40.42 & 0.01878 & 40.8 \\
\hline 35 & 81280 & 0.01938 & 1575 & 398370 & 2938107 & 36.15 & 0.02049 & 36.6 \\
\hline 40 & 79705 & 0.02366 & 1886 & 393296 & 2539737 & 31.86 & 0.02592 & 32.3 \\
\hline 45 & 77819 & 0.03302 & 2570 & 376907 & 2146441 & 27.58 & 0.03616 & 28.1 \\
\hline 50 & 75249 & 0.04830 & 3635 & 364615 & 1769534 & 23.52 & 0.04693 & 24.0 \\
\hline 55 & 71615 & 0.07686 & 5504 & 344431 & 1404919 & 19.62 & 0.07423 & 20.1 \\
\hline 60 & 66110 & 0.11384 & 7526 & 310825 & 1060488 & 16.04 & 0.11355 & 16.5 \\
\hline 65 & 58584 & 0.17934 & 10507 & 262234 & 749662 & 12.80 & 0.16995 & 13.2 \\
\hline 70 & 48078 & 1.00000 & 48078 & 487428 & 487428 & 10.14 & 1.00000 & 10.4 \\
\hline
\end{tabular}

West Bengal (2001-05) (Generated from SRS life tables of West Bengal)

\begin{tabular}{|c|c|c|c|c|c|c|c|c|}
\hline \multirow{2}{*}{ age } & \multicolumn{7}{|c|}{ Generated } & \multicolumn{2}{c|}{ SRS } \\
\cline { 2 - 9 } & $\mathrm{lx}$ & $\mathrm{qx}$ & $\mathrm{dx}$ & $\mathrm{Lx}$ & $\mathrm{Tx}$ & $\mathrm{e}_{\mathrm{x}}{ }^{0}$ & $\mathrm{qx}$ & $\mathrm{e}_{\mathrm{x}}{ }^{0}$ \\
\hline 0 & 100000 & 0.04667 & 4667 & 96621 & 6490000 & 64.9 & 0.04346 & 64.9 \\
\hline 1 & 95333 & 0.05768 & 5499 & 366194 & 6397226 & 67.1 & 0.05465 & 66.9 \\
\hline 5 & 89834 & 0.00614 & 552 & 447618 & 5957808 & 66.32 & 0.00514 & 66.7 \\
\hline 10 & 89283 & 0.00615 & 549 & 445794 & 5502296 & 61.63 & 0.00354 & 62 \\
\hline 15 & 88734 & 0.00769 & 682 & 447813 & 5056502 & 56.99 & 0.00623 & 57.2 \\
\hline 20 & 88051 & 0.00619 & 545 & 458627 & 4608689 & 52.34 & 0.00722 & 52.5 \\
\hline 25 & 87506 & 0.00898 & 786 & 419544 & 4150062 & 47.43 & 0.00866 & 47.9 \\
\hline 30 & 86721 & 0.00839 & 728 & 416783 & 3730518 & 43.02 & 0.00946 & 43.3 \\
\hline 35 & 85993 & 0.01469 & 1263 & 452156 & 3313735 & 38.53 & 0.01218 & 38.7 \\
\hline 40 & 84730 & 0.01716 & 1454 & 405189 & 2861579 & 33.77 & 0.01637 & 34.1 \\
\hline 45 & 83276 & 0.02482 & 2067 & 401986 & 2456390 & 29.5 & 0.02656 & 29.7 \\
\hline 50 & 81209 & 0.03869 & 3142 & 414969 & 2054404 & 25.3 & 0.03613 & 25.4 \\
\hline 55 & 78067 & 0.07200 & 5621 & 395838 & 1639435 & 21 & 0.06570 & 21.3 \\
\hline 60 & 72446 & 0.11101 & 8042 & 339496 & 1243596 & 17.17 & 0.10186 & 17.6 \\
\hline 65 & 64404 & 0.16263 & 10474 & 297470 & 904100 & 14.04 & 0.15275 & 14.2 \\
\hline 70 & 53930 & 1.00000 & 53930 & 606630 & 606630 & 11.25 & 1.00000 & 11.3 \\
\hline
\end{tabular}




\section{Gujarat (2001-05) (Generated from SRS life tables of Gujarat)}

\begin{tabular}{|c|c|c|c|c|c|c|c|c|}
\hline \multirow{2}{*}{ age } & \multicolumn{7}{|c|}{ Generated } & \multicolumn{2}{c|}{ SRS } \\
\cline { 2 - 9 } & $\mathrm{lx}$ & $\mathrm{qx}$ & $\mathrm{dx}$ & $\mathrm{Lx}$ & $\mathrm{Tx}$ & $\mathrm{e}_{\mathrm{x}}{ }^{0}$ & $\mathrm{qx}$ & $\mathrm{e}_{\mathrm{x}}{ }^{0}$ \\
\hline 0 & 100000 & 0.05788 & 5788 & 95809 & 6390000 & 63.9 & 0.06143 & 63.9 \\
\hline 1 & 94212 & 0.02223 & 2094 & 371218 & 6310173 & 66.98 & 0.03183 & 67.1 \\
\hline 5 & 92118 & 0.00609 & 561 & 459016 & 5941381 & 64.50 & 0.00628 & 65.2 \\
\hline 10 & 91557 & 0.00548 & 502 & 497442 & 5515749 & 60.24 & 0.00489 & 60.6 \\
\hline 15 & 91055 & 0.00784 & 714 & 431883 & 5018307 & 55.11 & 0.00668 & 55.9 \\
\hline 20 & 90341 & 0.00876 & 791 & 467835 & 4586424 & 50.77 & 0.00906 & 51.3 \\
\hline 25 & 89550 & 0.01086 & 973 & 441423 & 4118589 & 45.99 & 0.01104 & 46.7 \\
\hline 30 & 88577 & 0.01421 & 1258 & 426395 & 3677165 & 41.51 & 0.01321 & 42.2 \\
\hline 35 & 87319 & 0.01418 & 1238 & 438879 & 3250770 & 37.23 & 0.01563 & 37.7 \\
\hline 40 & 86081 & 0.01921 & 1654 & 415404 & 2811892 & 32.67 & 0.01981 & 33.3 \\
\hline 45 & 84427 & 0.02915 & 2461 & 435360 & 2396488 & 28.39 & 0.02724 & 28.9 \\
\hline 50 & 81966 & 0.04176 & 3423 & 372969 & 1961128 & 23.93 & 0.03840 & 24.6 \\
\hline 55 & 78543 & 0.06862 & 5390 & 399642 & 1588159 & 20.22 & 0.06324 & 20.5 \\
\hline 60 & 73154 & 0.10685 & 7816 & 339361 & 1188517 & 16.25 & 0.10285 & 16.7 \\
\hline 65 & 65337 & 0.17385 & 11359 & 307716 & 849156 & 13.00 & 0.15985 & 13.3 \\
\hline 70 & 53978 & 1.00000 & 53978 & 541440 & 541440 & 10.03 & 1.00000 & 10.4 \\
\hline
\end{tabular}
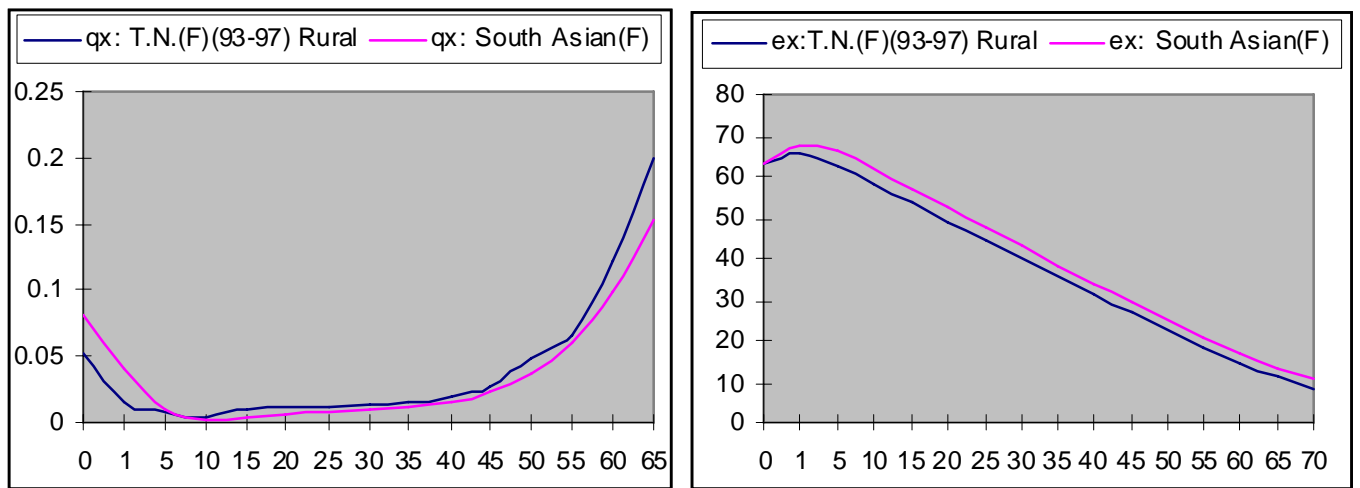

Figure 1: SRS (Female-Rural: 1993-97) and South Asian Model Life Tables (Interpolated) for Tamil Nadu (qx and ex portion): 

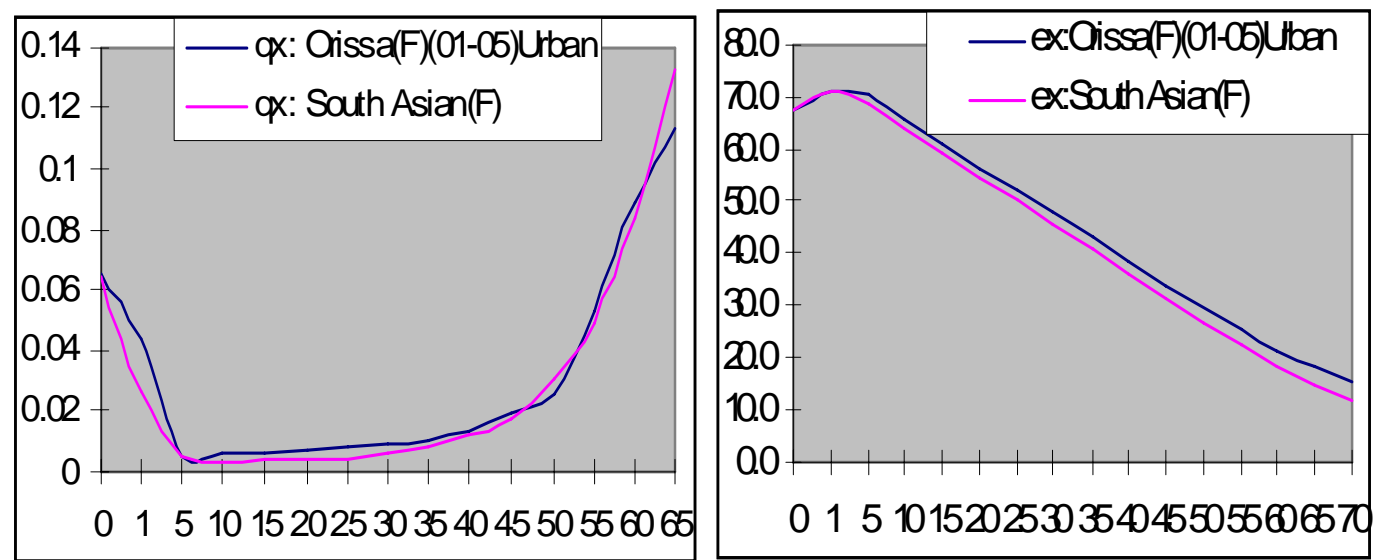

Figure 2: SRS (Female-Urban: 2001-05) and South Asian Model Life Tables (Interpolated) for Orissa:
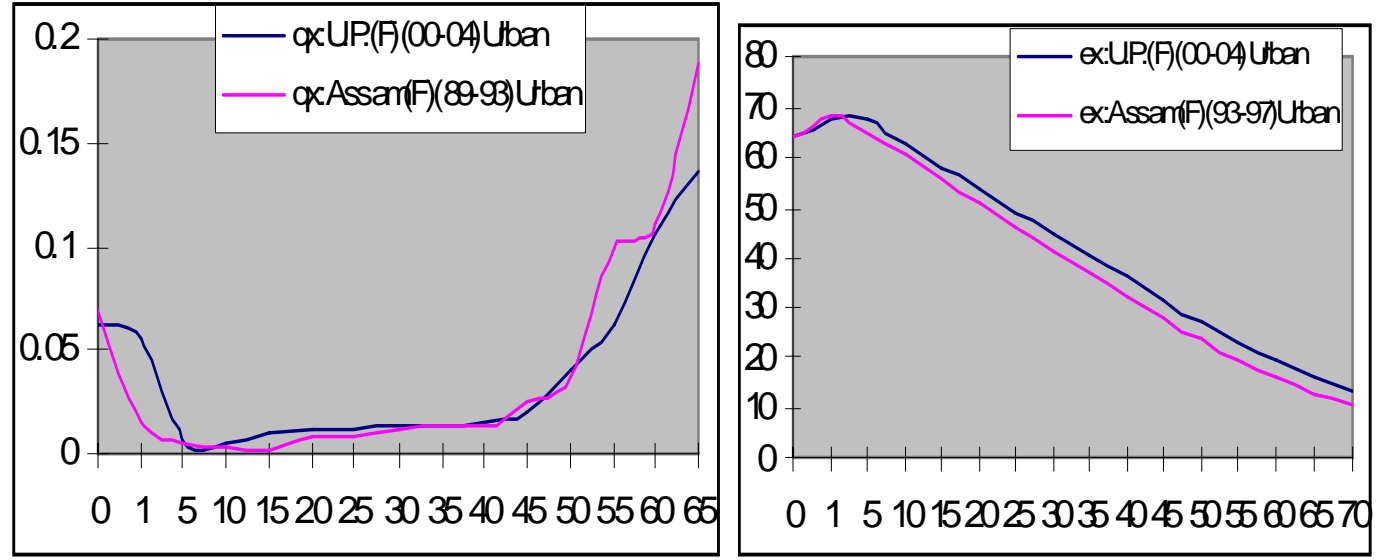

Figure 3: SRS Life Tables of U.P. (2000-04) and Assam (2001-05) for female (Urban):
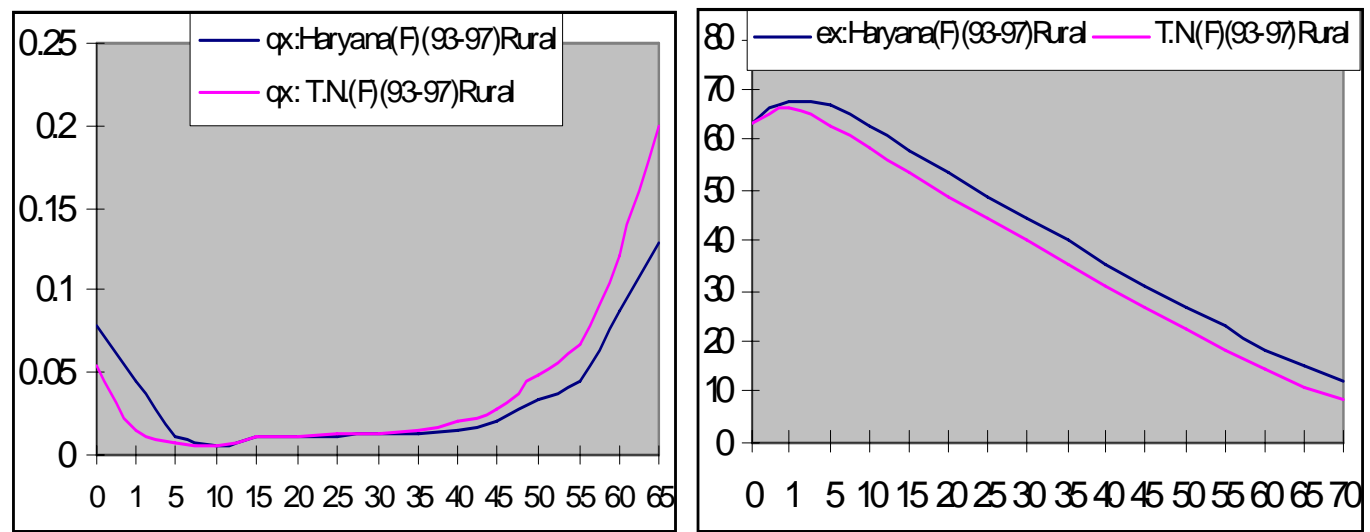

Figure 4: SRS Life Tables of Haryana and Tamil Nadu (1993-97) for Female (Rural): 

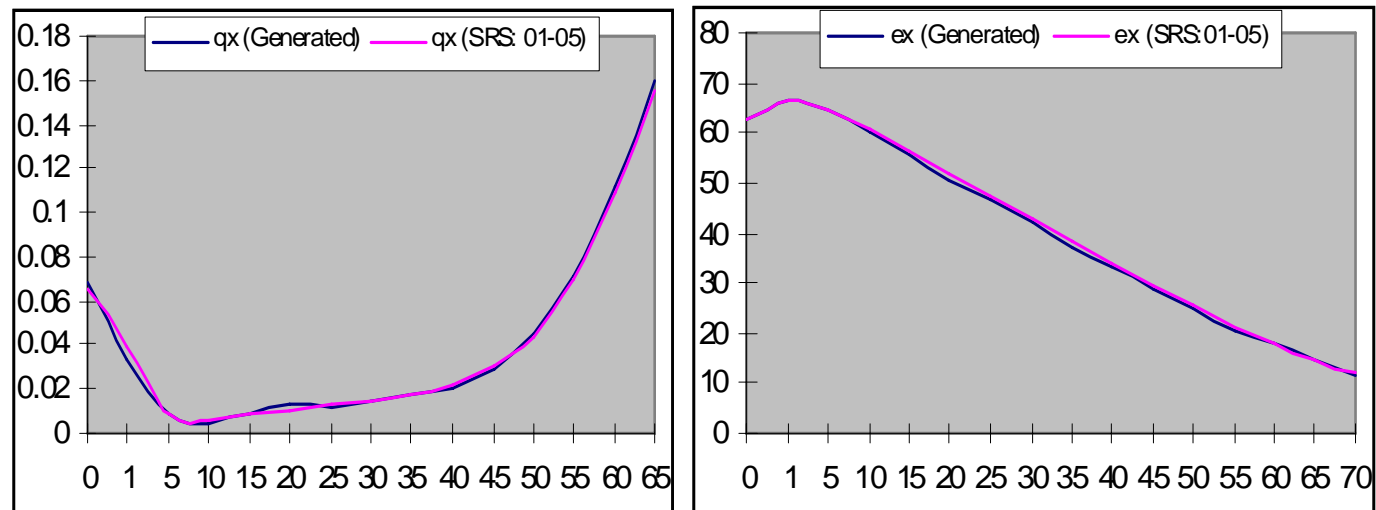

Figure 5: Generated and SRS Life Tables for India (2001-05) (Generated from SRS life tables of India) (qx and ex portion):
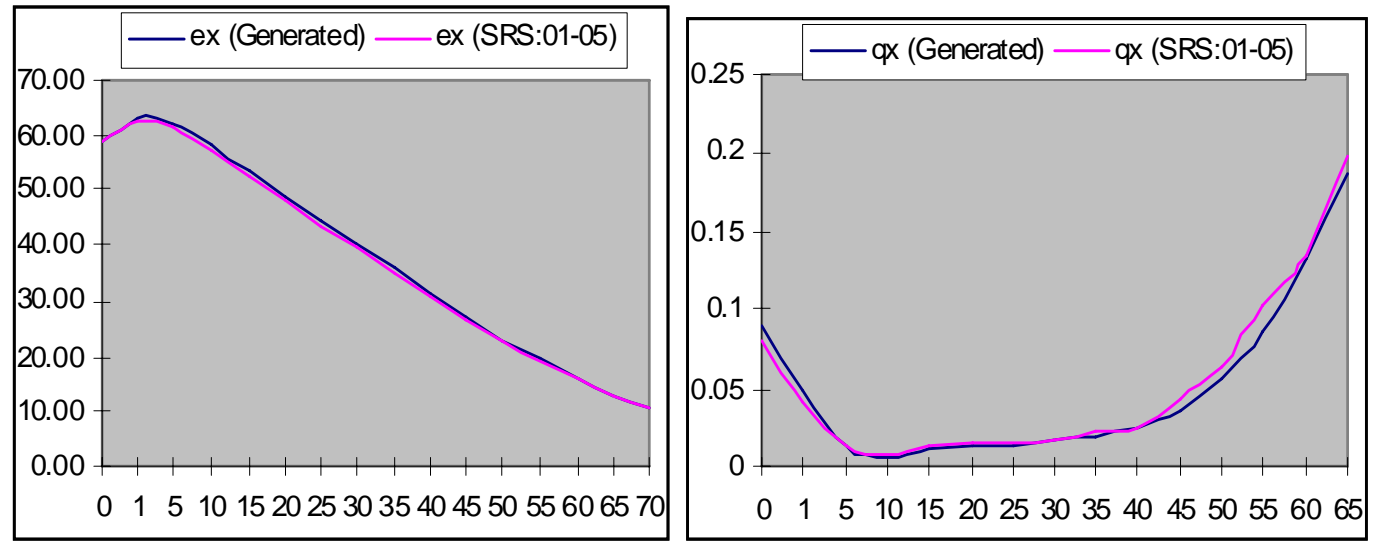

Figure 6: Generated and SRS Life Tables for Assam (2001-05) (Generated from SRS life tables of India) (qx and ex portion):
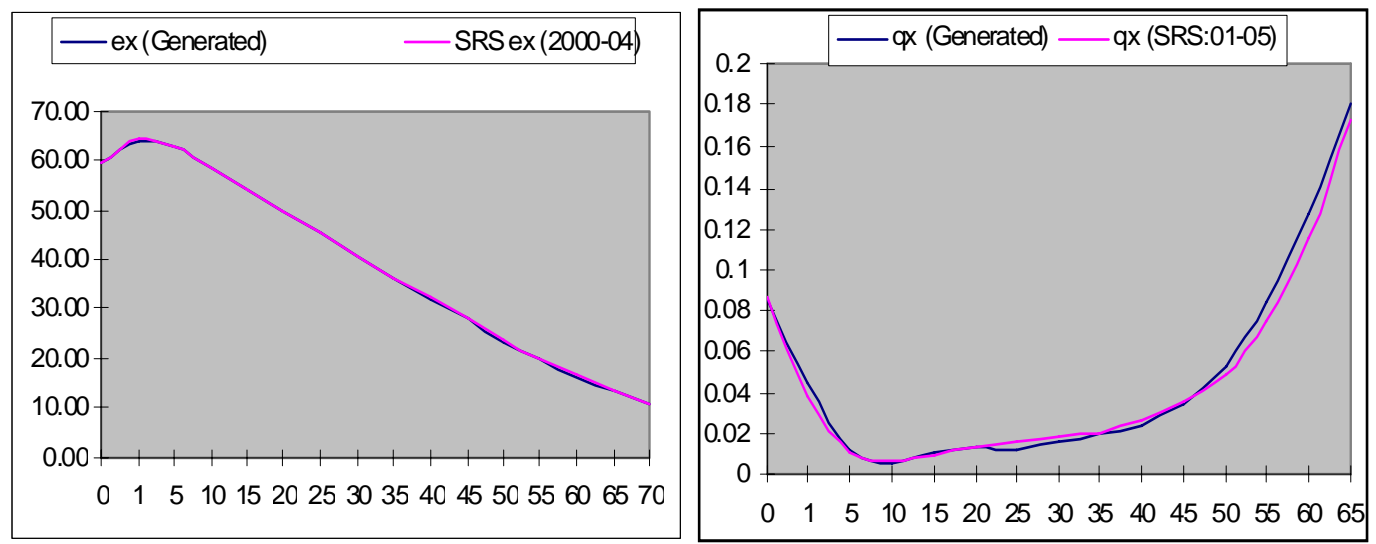

Figure 7: Generated and SRS Life Tables for Uttar Pradesh (2000-04) (Generated from SRS life tables of India) (qx and ex portion): 

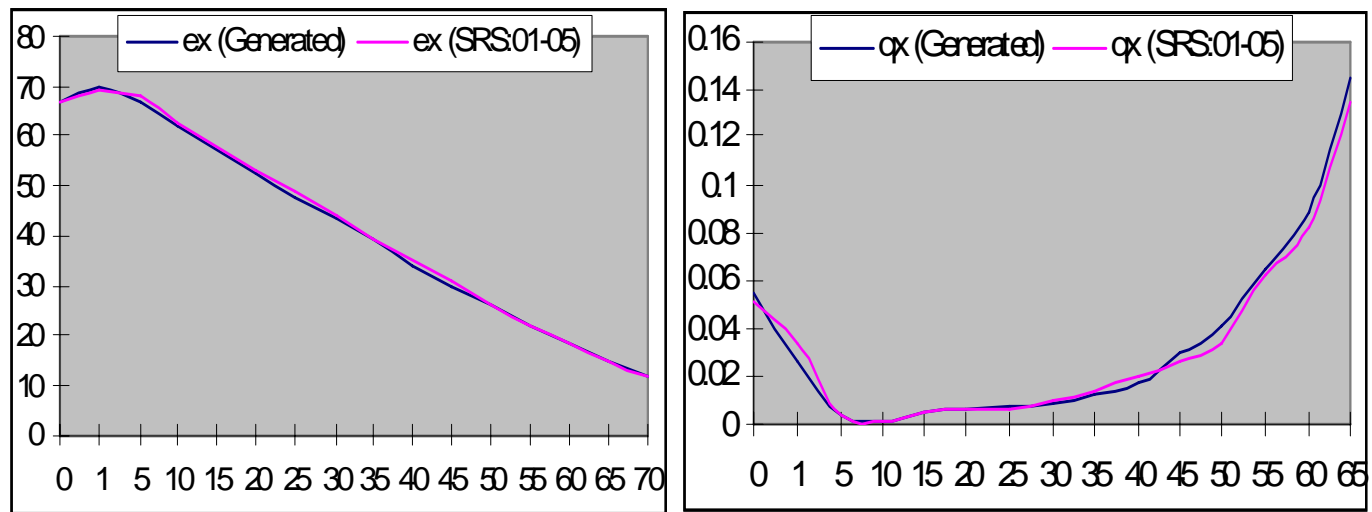

Figure 8(1): qx and ex portion of the Life Table of Himachal Pradesh (2001-05) (Generated from SRS life tables of Himachal Pradesh):
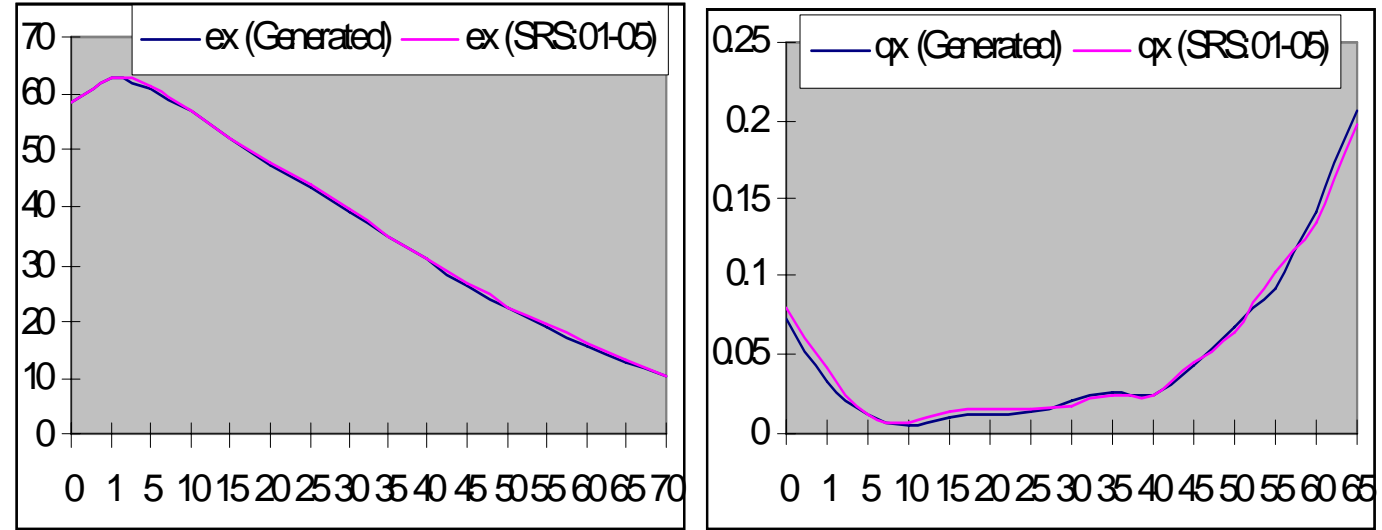

Figure 8(2): qx and ex portion of the Life Table of Assam (2001-05) (Generated from SRS life tables of Assam):
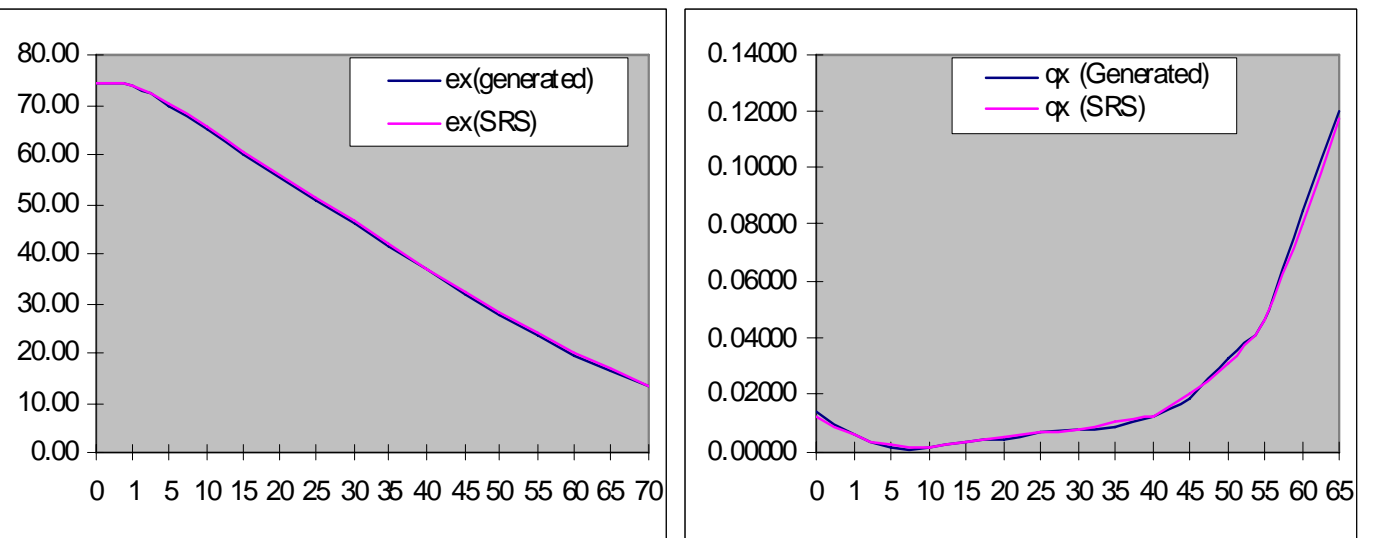

Figure 8(3): qx and ex portion of the Life Table of Kerala (2001-05) (Generated from SRS life tables of Kerala): 

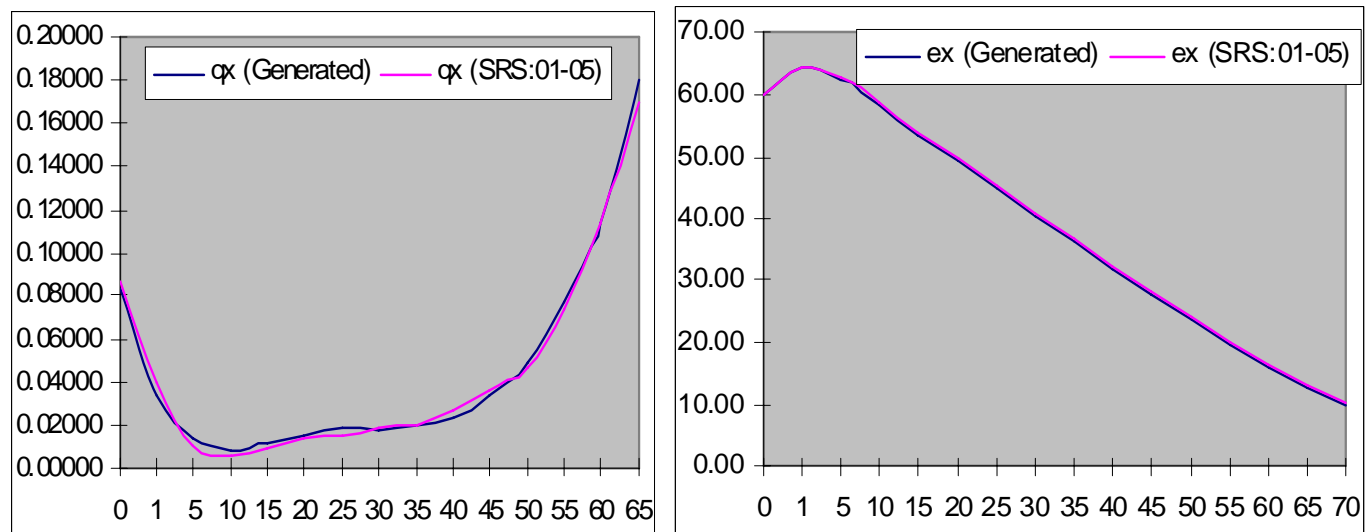

Figure 8(4): qx and ex portion of the Life Table of Uttar Pradesh (2001-05) (Generated from SRS life tables of Uttar Pradesh):
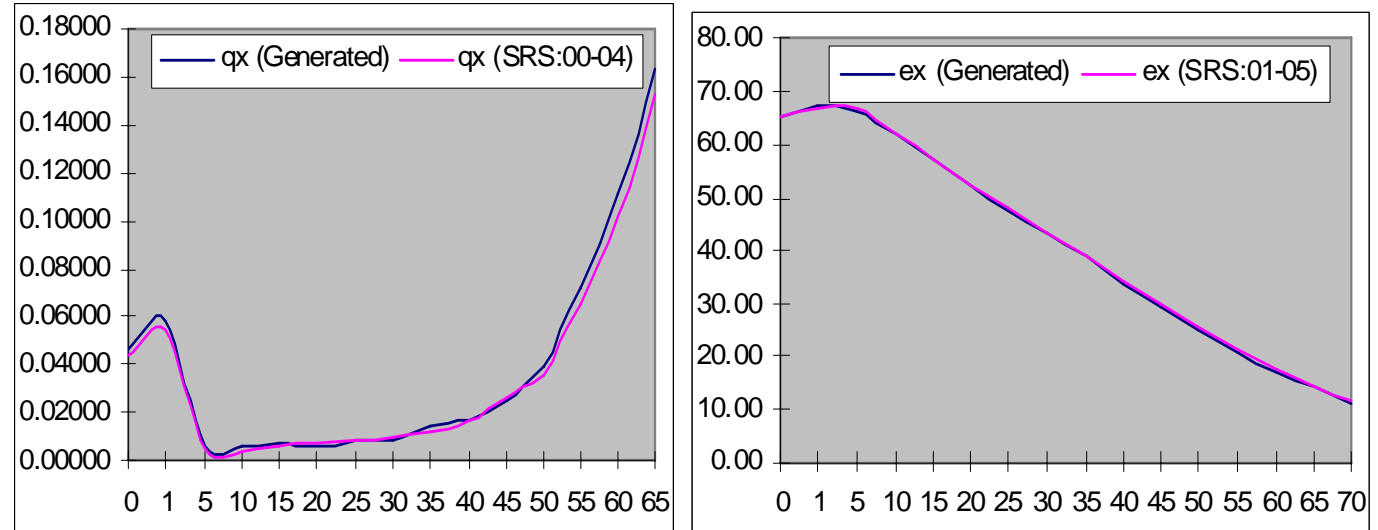

Figure 8(5): qx and ex portion of the Life Table of West Bengal (2001-05) (Generated from SRS life tables of West Bengal):
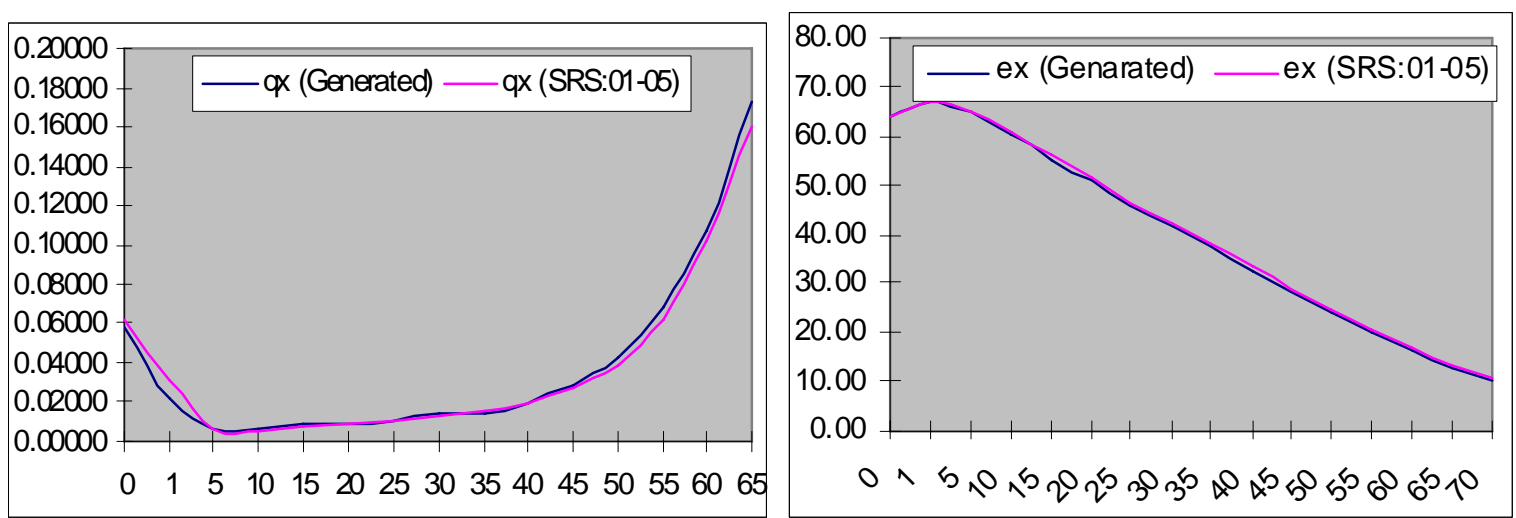

Figure 8(6): qx and ex portion of the Life Table of Gujarat (2001-05) (Generated from SRS life tables of Gujarat): 


\section{References}

[1] Shryock, H. S. and Seigel, J. S., 1976, Methods and Materials of Demography, Academic Press, New York.

[2] Swanson, D. A., 1989, "A State Based Regression Model for Estimating Sub State Life Expectancy”, Demography, 26(1), pp. 161-170.

[3] Bhat, P. N. M., 1987, "Mortality in India: Levels, Trends, and Patterns”, a dissertation in demography, Ann Arbor, Michigan: UMI.

[4] Malaker, C. R. 1986, "Estimation of Adult Mortality in India: 1971-81", Demography India, 15(1), pp. 126-135.

[5] United Nations, 1983, "Manual X: Indirect Techniques for Demographic Estimation”, Population Studies, no. 81, New York, USA.

[6] Sarma, R. and Choudhury L., 2010, "Indirect Estimation of Life expectancy at Birth at the District Level of India: A regression Approach”, $32^{\text {nd }}$ Annual Conference and National Seminar on Population and Public Health, IASP, Bhubaneswar.

[7] Government of India, 1970-75, 1976-80, 1986-90, 1987-91, 1989-93, 1990-94, 1991-95, 1992-96, 1993-97, 1996-00, 1997-01, 2000-04, 2001-05, SRS Based Abridged Life Tables, Registrar General, New Delhi.

[8] Adekola, O. A., 2001, “A Note on Life Expectancy in a Non-Homogeneous Population”, Journal of Operational Research Society, 52, pp. 842-843.

[9] Coale, A. J. and Demeney, P., 1983, Regional Model Life Tables and Stable Populations, Second edition, Academic Press, New York. 